\section{Eyeing new uses for protease inhibitors}

\author{
By Michael J. Haas, Senior Writer
}

A paper in the Journal of Clinical Investigation indicates that HIV protease inhibitors could be repurposed as neuroprotectants to block apoptosis in age-related macular degeneration, diabetic retinopathy and other conditions involving neuronal apoptosis. ${ }^{1}$ The findings add to mounting evidence demonstrating that HIV protease inhibitors can treat indications beyond HIV_-including cancer.

Companies contacted by SciBX agreed that the compounds' proven safety profiles made them promising candidates for repurposing in a range of non-HIV indications.

Indeed, according to Guido Kroemer, coauthor on the JCI paper, "the neuroprotective effects of HIV-1 protease inhibitors are broad. Other possible indications include neonatal hypoxia, brain trauma and spinal injury. All of these situations are accompanied by sudden, massive neuronal apoptosis."

Kroemer, a research director at Institut National de la Santé et de la Recherche Médicale (INSERM), also suggested that protease inhibitors could inhibit apoptosis in slowly neurodegenerative diseases like

Table 1. Outside HIV. Recent findings published in the Journal of Clinical Investigation suggest that HIV protease inhibitors could be used to inhibit or prevent apoptosis in retinal detachment. A previous research team, which included two of the same researchers as the current one, demonstrated a similar antiapoptotic effect for HIV protease inhibitors in stroke. Thus the current team speculates that HIV protease inhibitors could inhibit neuronal apoptosis in other ophthalmic and neurodegenerative conditions. But they cautioned that disease-specific studies are needed to prove this, especially for gradually neurodegenerative conditions in which the side effects of long-term HIV protease inhibitor use might outweigh the benefits.

\section{Potential indications}

Age-related macular degeneration (AMD)

Alzheimer's disease (AD)

Brain trauma

Diabetic retinopathy

Neonatal hypoxia

Parkinson's disease (PD)

Retinal detachment

Spinal injury

Stroke
Parkinson's disease (PD) and Alzheimer's disease (AD; see Table 1, "Outside HIV").

The research team included scientists from Harvard Medical School, Kyushu University, the Mayo Clinic, the Institute Gustave-Roussy and Paris-Sud University. The team was led by Joan Miller, chair of ophthalmology at Harvard Medical School and chief of ophthalmology at the Massachusetts Eye and Ear Infirmary.

\section{Caspase puzzle}

HIV protease inhibitors were first postulated to have an antiapoptotic effect in humans in 1999, when it was reported that HIV patients treated with the drugs recovered normal levels of $\mathrm{CD} 4^{+} \mathrm{T}$ cells even when the treatment had no effect on viral loads. That led to speculation that protease inhibitors prevent $\mathrm{T}$ cell apoptosis. ${ }^{2,3}$ Since then, protease inhibitors have been shown to have antiapoptotic effects on nonimmune cells in models of sepsis, hepatitis and stroke. ${ }^{4,5}$ Paradoxically, also in 1999, HIV protease inhibitors were also found to have proapoptotic effects on cancer cells. ${ }^{6}$

The pro- and antiapoptotic mechanisms are not completely understood. But in both cases it is clear the compounds do not inhibit the target viral protein.

In cancer cells, HIV protease inhibitors have been shown to induce apoptosis by inhibiting the phosphorylation of protein kinase B (Akt). ${ }^{7,8}$ Nelfinavir has proven especially potent in this regard ${ }^{9,10}$ and has been the focus of at least two recent Phase I trials in cancer patients-one conducted by the National Cancer Institute and the other by a joint team from Friedrich-Alexander University of Erlangen-Nuremberg and the University of Oxford. ${ }^{9,11}$

The compounds' antiapoptotic mechanism of action has presented more of a puzzle.

Mitochondrial apoptosis-the main apoptotic pathway in mammalian cells-involves the activation of caspases, a family of cysteine proteases. Thus it was thought that HIV protease inhibitors acted by blocking a caspase-dependent pathway of apoptosis. But inhibition of caspase activation does not always translate to complete inhibition of apoptosis ${ }^{12}-\mathrm{a}$ fact that led the Miller team to speculate that HIV protease inhibitors might also target a caspase-independent pathway.

Using a mouse model of retinal detachment as well as murine retinal cell cultures, the team showed that a combination of two protease inhibitors marketed for HIV-ritonavir and nelfinavir-blocked both caspasedependent and caspase-independent pathways of apoptosis.

The drugs acted by inhibiting mitochondrial outer membrane permeabilization (MOMP). That prevented the release of cytochrome $c$ (CYCS) and apoptosis-inducing factor (AIF), which trigger the caspasedependent and caspase-independent pathways, respectively.

The study did not determine specific mitochondrial targets of the protease inhibitors.

Abbott Laboratories markets Norvir ritonavir. Pfizer Inc. markets Viracept nelfinavir in the U.S., Canada and Japan; Roche markets the drug elsewhere.

The researchers also found that AIF may induce the release of CYCS 
from mitochondria, suggesting that cross-talk between the pathways allows apoptosis to proceed even when one pathway is blocked by a caspase inhibitor.

Miller told SciBX the team's results reconcile the apparent contradictions between previous studies by connecting new pieces to existing ones in the mechanistic puzzle.

\section{A closer look}

Company representatives contacted by SciBX agreed that the JCI results, combined with the well-known pharmacokinetic and safety profiles of protease inhibitors, made the drugs highly promising neuroprotectants. But they added that further studies are needed to clarify the compounds' mechanisms of action and confirm their antiapoptotic effects in humans.

According to David Bearss, VP and chief scientist at SuperGen Inc., "many papers have shown that protease inhibitors affect apoptosis, which is not surprising because apoptotic pathways often involve proteases."

He added: "The researchers did these experiments in cell-based systems, which clarifies the pathways involved. But biochemical assays could identify the specific molecules involved" and determine exactly how protease inhibitors block the release of both CYCS and AIF from mitochondria.

"There may be a mitochondrial protein whose role is not known, and a biochemical assay could help elucidate it." Bearss said.

SuperGen has two protease programs for cancer in lead optimization.

One program targets Site- 1 protease (S1P) and S2P to treat pancreatic cancer. The proteases regulate cholesterol biosynthesis but are also upregulated in several tumor types. The other program targets transmembrane protease, serine 2 (TMPRSS2) to treat prostate cancer. TMPRSS 2 is overexpressed in about $40 \%$ of prostate cancer patients and is upregulated by androgenic hormones in prostate cancer cells.

Bearss said preclinical studies in both programs are planned for this year.

Merck \& Co. Inc.'s Donald Nicholson agreed with Bearss that the JCI paper adds "further evidence to the growing body of scientific data showing, unexpectedly, that HIV protease inhibitors are able to suppress pathogenic cell death in mammalian cells." Nicholson is VP of franchise worldwide basic research (head, bone, respiratory, immunology \& endocrine franchise).

He likewise noted the mechanism of action for HIV protease inhibitors in the new indications remains unclear. Based on the results reported in JCI, he suggested that HIV protease inhibitors might act on multiple targets in the mitochondrion to protect neural cells.

"The critical question, of course, is whether similar benefits can be seen in humans and whether these inhibitors might find therapeutic application in serious neurodegenerative disorders," Nicholson said.

Merck markets the HIV protease inhibitor Crixivan indinavir to treat HIV.

\section{Wider indications}

Other companies were interested in the range of specific neurodegenerative indications that HIV protease inhibitors might treat.

"The identification of agents that could inhibit photoreceptor apoptosis would be a major medical breakthrough," said Tom Hohman, VP of retinal pharmaceutical development at Alcon Laboratories Inc.

In addition to treating retinal detachment, as reported in the JCI paper, Hohman said photoreceptor apoptosis is characteristic of geographic atrophy, an advanced form of AMD. "Very little is known about the underlying disease pathophysiology" of geographic atrophy, though variations in complement factor genes and oxidative damage to cell membranes have both been implicated, he said.

Hohman added that although clinical trials "of antioxidants or complement factor inhibitors are either planned or in progress, therapeutics based on these mechanisms of action are far in the future." Thus, "agents that block mitochondrial pathways of apoptosis may be an alternative treatment" for geographic atrophy.

Alcon's Retaane anecortave is in Phase III trials to treat wet AMD and to reduce the risk of conversion of dry AMD to wet AMD. In 2007, the company received an FDA approval letter for an NDA for Retaane to treat wet AMD that requested an additional trial.

In April, Novartis AG announced plans to acquire a 25\% stake in Alcon from Nestle S.A.

"Protection of the host cells in neurodegenerative disease is critical because once cells have died there is no drug that will bring them back to life," noted Erik Miljan, head of stem cell discovery at ReNeuron Group plc. "From a commercial perspective, what makes the JCI finding so exciting is that HIV protease inhibitors appear to protect cells from a broad range of apoptotic insults."

Miljan added that neuronal apoptosis "doesn't happen immediately. It can occur over days and weeks following an acute insult," such as spinal injury or brain trauma. "This secondary damage could be managed with the antiapoptotic activity of HIV protease inhibitors. You wouldn't be able to regenerate cells, but you could prevent degeneration," he said.

Thus, Miljan was especially interested in the potential use of HIV protease inhibitors in indications where gradual neurodegeneration is associated with neuronal apoptosis, such as $\mathrm{PD}, \mathrm{AD}$, Huntington's disease (HD) and amyotrophic lateral sclerosis (ALS).

ReNeuron has human stem cell lines (ReN004) in discovery research for PD.

"The use of HIV protease inhibitors would not be a silver bullet or cure, but it could stop disease progression in its tracks" in these indications, said Miljan.

Moreover, he said the JCI paper demonstrates that HIV protease inhibitors have a broader spectrum of activity-and therefore might be more effective-than earlier antiapoptotic neuroprotectants, which ultimately proved ineffective in the clinic due to adverse effects, dosing problems and low activity. He cited the discontinuation of trials of the antiapoptotic agents TCH346 and CEP-1347 as cases in point.

In late 2004, Novartis discontinued a Phase II trial of TCH346 to treat ALS when the study showed no effect on the rate of progression or survival. The company had stopped an earlier Phase II trial in PD for similar reasons. The compound targeted glyceraldehyde 3-phosphate dehydrogenase (GAPDH). 


\section{TARGETS \& MECHANISMS}

Table 2. HIV protease inhibitors. There are about nine HIV protease inhibitors on the market. Except for Merck \& Co. Inc.'s MK-8122, not many new protease inhibitors appear to be in clinical development. Merck (NYSE:MRK) obtained exclusive worldwide rights to MK-8122 from Ambrilia BioPharma (TSX:AMB) in 2006. The compound is in Phase I studies.

\begin{tabular}{ll}
\hline Company & Drug \\
\hline Abbott Laboratories (NYSE:ABT) & $\begin{array}{l}\text { Kaletra lopinavir/ } \\
\text { ritonavir }\end{array}$ \\
\hline Abbott & Norvir ritonavir \\
\hline Boehringer Ingelheim GmbH & Aptivus tipranavir \\
\hline Bristol-Myers Squibb Co. (NYSE:BMY) & Reyataz atazanavir \\
\hline Johnson \& Johnson (NYSE:JNJ) & Prezista darunavir \\
\hline Merck & Crixivan indinavir \\
\hline Pfizer Inc. (NYSE:PFE) & Viracept nelfinavir \\
\hline Roche (SWX:ROG) & Invirase saquinavir \\
\hline Vertex Pharmaceuticals Inc. (NASDAQ:VRTX)/ & $\begin{array}{l}\text { Lexiva fosamprenavir } \\
\text { (Telzir) }\end{array}$ \\
GlaxoSmithKline plc (LSE:GSK; NYSE:GSK) &
\end{tabular}

In 2005, partners Cephalon Inc. and H. Lundbeck A/S discontinued a Phase II/III study of CEP-1347 to treat PD because final data were not expected to provide evidence of a significant treatment effect. That compound targeted the mixed lineage kinase (MLK) family.

According to Miljan, setbacks like these led neurodegeneration companies to conclude that progression of neurodegenerative diseases could not be slowed by blocking apoptosis.

But the JCI paper, he said, suggests an alternative explanation.

"Maybe the original drugs just weren't effective" but the antiapoptotic approach itself is sound, Miljan said. "The mode of action of HIV protease inhibitors in this capacity opens a new avenue" and might renew interest in antiapoptotic approaches to treating neurodegenerative disorders.

Miljan wanted to know whether there was any correlation between the use of HIV protease inhibitors and decreased incidence of neurodegenerative disorders among HIV patients.

\section{Prospects look good}

Both Miljan and Bearss suggested companies with HIV protease inhibitors would not encounter serious difficulties in repurposing the drugs for neurodegenerative indications because the compounds have well-known pharmacokinetic and safety profiles.

"Nelfinavir and ritonavir have been used to treat millions of patients for HIV," Miljan noted.

The range of possible neurodegenerative indications in which protease inhibitors could be effective "represents a real market," he added. "What's the harm in trying? The side effects are already known."

In the U.S. there are at least nine marketed protease inhibitors for HIV (see Table 2, "HIV protease inhibitors"). Their side effects can include increased hyperlipidemia and atherosclerosis.
Because these side effects could outweigh the benefits with long-term use, Miller plans to focus on short-term use of protease inhibitors as neuroprotectants in acute indications-especially ophthalmic indications, which is her primary area of interest.

Going forward, the research team plans to tease out more of the mechanism in ophthalmic indications and determine how best to take protease inhibitors into clinical trials for retinal degeneration in AMD, Miller said.

"If you could combine a neuroprotectant with an antiangiogenic compound, you could get better results" in preventing retinal degeneration than with an antiangiogenic alone, she said.

Miller said there are no immediate plans to patent the findings reported in JCI.

\section{REFERENCES}

1. Hisatomi, T. et al. J. Clin. Invest.; published online May 22, 2008; doi:10.1172/JCl34267

Contact: Joan W. Miller, Harvard Medical School, Boston, Mass. e-mail: joan_miller@meei.harvard.edu

Contact: Guido Kroemer, Institut National de la Santé et de la Recherche Médicale (INSERM), Villejuif, France

e-mail: kroemer@igr.fr

2. Staszewski, S. et al. N. Engl. J. Med. 341, 1865-1873 (1999)

3. Deeks, S. \& Grant, R. Antivir. Ther. 4, 7-11 (1999)

4. Rizza, S. \& Badley, A. Med. Chem. 4, 75-79 (2008)

5. Weaver, J. et al. J. Clin. Invest. 115, 1828-1838 (2005)

6. Niehues, T. et al. AIDS 13, 1148-1149 (1999)

7. Gupta, A. et al. Neoplasia 9, 271-278 (2007)

8. Plastaras, J. et al. Cancer Biol. Ther. 7(5); published online Feb. 14, 2008

9. Gills, J. et al. Autophagy 4, 107-109 (2008)

10. Gills, J. et al. Clin. Cancer Res. 13, 5183-5194 (2007)

11. Brunner, T. et al. J. Clin. Oncol. 26, 2699-2706 (2008)

12. Green, D. \& Kroemer, G. J. Clin. Invest. 115, 2610-2617 (2005)

\section{COMPANIES AND INSTITUTIONS MENTIONED}

Abbott Laboratories (NYSE:ABT), Abbott Park, III.

Alcon Laboratories Inc. (NYSE:ACL), Fort Worth, Texas

Cephalon Inc. (NASDAQ:CEPH), Frazer, Pa.

Friedrich-Alexander University of Erlangen-Nuremberg, Nuremberg, Germany

H. Lundbeck A/S (CSE:LUN), Copenhagen, Denmark

Harvard Medical School, Boston, Mass.

Institut National de la Santé et de la Recherche Médicale, Villejuif,

France

Institute Gustave-Roussy, Villejuif, France

Kyushu University, Fukuoka, Japan

Massachusetts Eye and Ear Infirmary, Boston, Mass.

Mayo Clinic, Rochester, Minn.

Merck \& Co Inc. (NYSE:MRK), Whitehouse Station, N.J.

National Cancer Institute, Bethesda, Md.

Nestle S.A. (SWX:NESN), Vevey, Switzerland

Novartis AG (NYSE:NVS; SWX:NOVN), Basel, Switzerland

Paris-Sud University, Villejuif, France

Pfizer Inc. (NYSE:PFE), New York, N.Y.

ReNeuron Group plc (LSE:RENE), Guildford, U.K.

Roche (SWX:ROG), Basel, Switzerland

SuperGen Inc. (NASDAQ:SUPG), Dublin, Calif.

University of Oxford, Oxford, U.K. 